\title{
IMPROVED STAND FOR THE DONOR EYE FOR CORNEAL GRAFTING*
}

BY

\section{MAURICE H. LUNTZ}

St. Mary's Hospital (Western Ophthalmic Hospital) London

A STAND, developed at this hospital, for holding the donor eye while a corneal graft is trephined and dissected is particularly useful for lamellar, annular, or segmental grafts, especially where direct sutures are used (Fig. 1).

Essentially this is a modified Tudor-Thomas stand, so designed as to rotate on a plinth carrying a spring-loaded metal ball (Fig. 2A), which engages consecutively into four notches placed at $90^{\circ}$ intervals at the bottom of the stand (Fig. 2B). By this means the stand can be engaged at four positions $90^{\circ}$ apart during one complete revolution. Disengagement from a "stop" requires no effort so that the entire mechanism works smoothly and efficiently. The plinth also has a pair of handles (Fig. 2C, c) with separate "lifters" for lifting it out of the sterilizer.

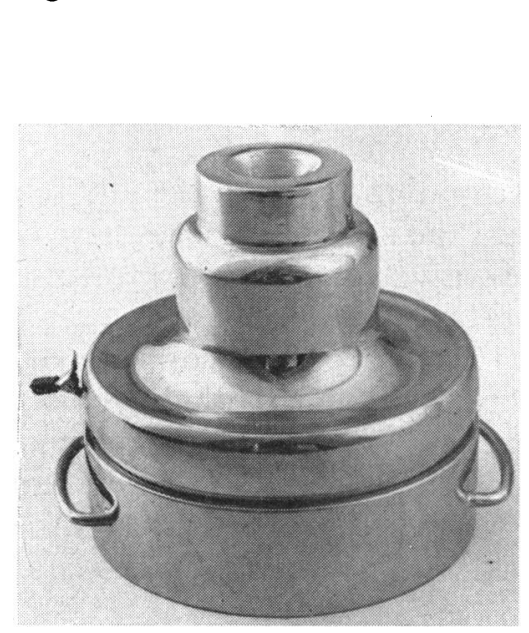

Fig. 1.-Complete stand assembled.

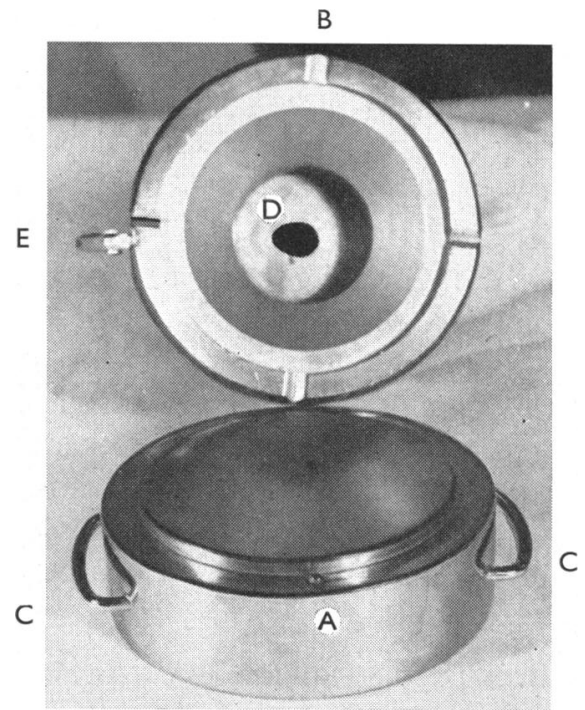

FIG. 2.-Plinth dismantled, showing spring ball (A), notches (B), handles (C), eccentric hole (D), and clip for suture $(\mathrm{E})$.

Three new features are embodied in this instrument:

(a) The free rotation of the modified Tudor-Thomas stand on the plinth facilitates the dissection of a lamellar, annular, or segmental graft. The 
stand will engage the metal ball on the base in four positions $90^{\circ}$ apart, so that the insertion of four direct sutures into the graft, accurately placed at right angles, becomes a simple matter.

(b) The hole in the stand which takes the optic nerve and fixation suture has been eccentrically placed (Fig. 2D), so that the cornea is vertical when the donor eye is fixed on the stand. This facilitates both dissecting the graft and placing the sutures.

(c) A clip (Fig. 2E) attached to the stand will hold the fixation suture. Occasionally the use of this clip will not produce adequate tension in the donor eyeball so that it becomes difficult to trephine the cornea. In this case the necessary tension can be got by direct traction on the fixation suture with a Spencer-Wells forceps while the surgeon trephines. The fixation suture is then fixed in the clip for the rest of the procedure.

It is felt that this has advantages over the original Tudor-Thomas stand, and that it may become a useful instrument in keratoplastic procedures.

I am indebted to John Weiss and Son Ltd., London, for making the stand and for their patience during the trial and error period. 